\title{
Hemorragia uterina irregular de origen funcional ${ }^{(*)}$
}

\author{
Doctor Edwăn C. Hamblen (**)
}

(Traducción y resumen del doctor G. Echeverry P.)

El término hemorragia funcional, significa para nosotros que hemos llegado a un diagnóstico de exclusión y por conisiguiente que hemos eliminado todas las causas-médicas, ginecológicas o enciocrinas que pueden producir tal hemorragia. Consideramos ia nemorragia uterina funcional como el resultado de alguna irregularidad en las relaciones hipotálamo-pituitaria-ovario; las enfermedades endocrinas puras que producen hemorragia uterina, tales como ciertos estados de hipotiroidismo, tumores endocrinos del ovario, etc., no constituyen hemorragia funcional en nuestro concepto.

En la mayoria de las adolescentes que presentan irregularidades en sus menstruaciones, debe asumirse una causa funcional, a no ser que se demuestre que tienen un embarazo complicado, un tumor ovárico o una discrasia sanguínea grave, especialmente del tipo del púrpura hemorrágico. En mujeres casadas jóvenes, debemos ante todo excluir el embarazo como causa de hemorragia irregular, y en las mujeres de más de veinticinco años practicar la dilatación y curetaje diagnósticos, antes de asumir que la hemo. rragia es funcional. Así mismo debe eliminarse cuidadosamente la existencia de miomas submucosos.

A) tratar de las irregularidades de la hemorragia uterina hacemos dos grandes categorías: las deficiencias en el flujo de san.

(*) Conferencia dictada por el Profesor E. C. Hamblen, en la Facultad de Medicina Javeriana en febrero de 1959 y publicado con su permiso.

(**) Profesor de endocrinología de Duke University. Durham, U. S. A. 
gre y los excesos. Esto con el fin de evitar el uso de términos como hipermenorrea, oligomenorrea, menorragia, metrorragia, etc., usados por diversas personas en diferentes sentidos, y que se prestan a confusión. Cuando hablamos de deficiencias en el flujo sanguíneo incluímos las pacientes que, o sangran en muy poca cantidad, o con muy poca frecuencia, o por periodos de tiempo muy cortos. Cuando hablamos de excesos, incluímos las que sangran muy frecuentemente, o muy abundantemente, o por períodos de tiempo muy largos. Con términos tan simples como éstos, vamos a las base's elementales del problema, y evitamos confusiones.

Me parece oportuno revisar un poco algunos de los mecani: mos del ciclo ovárico normal o anormal, de acuerdo con el diagrama de Markee. La preparación del folículo para la ovulación está regida por la hormona folículo-estimulante del lóbulo antezior de la hipófisis, o FSH, pero esta hormona no tiene efecto sobre el folículo hasta tanto que éste no haya alcanzado un cierto grado de madurez. Entonces obra el FSH sobre el folículo para llevarlo al estado de ovulación, en asocio de la hormona luteinizante o LH, que es la que llamamos hormona ovulatoria. Cuando se produce la ovulación hay liberación inmediata de la gonado. trofina luteotrópica, que es la que estimula la formación y funrión del cuerpo lúteo.

Sabemos que la acción de FSH sobre el folículo no es por sí sola capaz de condicionar la producción de estrógenos, y que se requiere indispensablemente una secreción básica de LH durante este tiempo, para que las células tecales secreten los estrógenos. Después de la ovulación, las células tecales transformadas en células teca-luteínicas, continúan la producción de estrógenos, e ini cian la de progesterona, estimuladas por la secreción de hormon! iuteotrópica.

Entonces en ningún momento durante el ciclo menstrual los riveles estrogénicos bajan a cero. La mujer menstrúa o sangra cuando los niveles sanguíneos de estrógenos caen aproximadamente al $50 \%$ de los valores máximos. Inmediatamente después de la ovulación hay una ligera depresión en los niveles estrogé. nicos, pero éstos se recuperan en seguida, tan pronto como el cuerpo lúteo inicia su función normal. Esta depresión es, pues, caracteristica de los ciclos ovulatorios. Sie nota también en los ciclos normales una pequeña alza del nivel de estrógenos hacia el cuarto día después de iniciada la menstruación. Esta elevación, que en mi concepto es la que produce la hemostasis y la suspensión de la 
hemorragia menstrual, parece ser debida a producción autónoma de estrógeno por el ovario, ya que el FSH no inicia su est'mulo sino después de que el nuevo óvulo ha ll:gado a un cierto estado de progreso.

De aquí hemos deducido la siguiente teoría en relación con el mecanismo de estos fenómenos: los estrógenos son la hormona del crecimiento del endometrio; mientras el endometrio esté creciendo, estimulado por los estrógenos, no sangra; cuando cesa de crecer, por disminución de los estrógenos, comienza a regresar y si la regresión es brusca, sangra. Por esta razón podemos suspencer la hemorragia uterina en una rnujer cuando le suministra. mos estrógenos, y el efecto hemostático de éstos se hará presente in el curso de unos tres cías. Si a una mujer se le dan estrógenos por vía oral durante veinte días y se suspenden bruscamente, pasará también un espacio de tres o cuatro días antes de que venga la sangría de supresión.

Muchos de los casos de menstruaciones muy prolongadas se deben a que faita el alza estrogénica hemostática post-menstrual. Igualmente, cuando la baja moderada y transitoria de estrógenos que vimos que hay normalmente después de la ovulación, se con-vierte en una caída crítica de los estrógenos hasta un 50\% de $10 \mathrm{~s}$ niveles normales, se ocasiona la hemorragia de ovulación o hemorragia bifásica, la cual continuará hasta tanto el nivel estrogéni. co no recupere sus cifras normales.

Muchos de los ciclos cortos, de menos de 25 d'as, se deben a que, después de la ovulación, la función del cuerpo lúteo se hace por muy poco tiempo, dando una fase luteal corta. Así, la menstruación se produce a partir de un endometrio progestacional inmaduro, lo cual es peligroso desde el punto de vista de un embarazo, pues al sobrevenir éste anidaría sobre un endometrio que va a comenzar su regresión, en el momento de la nidación.

Cuando los niveles estrogénicos son persistentemente altos y no hay ovulación, el endometrio va proliferando continuamente hasta hacerse hiperplásico. Si por cualquier razón hay una baja brusca de los estrógenos la paciente comenzará a sangrar persistentemente a partir de un endometrio que no ha entrado en fase secretora y que por consiguiente no tiene una descamación ade. cuada. Por esta razón, en estos casos, es mejor no tratar de hacer la hemostasis con estrógenos para no aumentar la hiperplasia, sino dar progesterona con el objeto de obtener una descamación 
àdecuada del endometrio, y luego sí comenzar los ciclos estrógeno-progesterona.

Las deficiencias en la menstruación son de mucha menor importancia clínica. Cuando son el resultado de una enfermedad méäica general o endocrina definida, el tratamiento se hará de acuerao con la causa. Si no se encontraren causas definidas podrán dejarse sin tratamiento, a no ser que el impacto psicológico sobre la paciente sea muy grande, es decir, en los casos en que las mu. jeres adquieren una preocupación exagerada por la poca frecuencia o escasez de sus reglas, y tienen la idea de que sin una sangria mensual abundante su salud puede estar comprometida. En ia misma forma, en los casos de infertilidad deben siempre tratarse de corregir estas deficiencias, investigando a fondo la paciente, y sobre todo tratando de precisar si hay o no ovulación, para buscar la corrección de todas estas definiciones y por cor. siguiente de la infertilidad. El tratamiento de los ciclos anovula. torios lo veremos en posterior conferencia. En las mujeres que tienen dos o tres ciclos ovulatorios al año, no debemos hacer trata. miento, porque sus funciones ováricas son normales y la hormonoterapia podría causar más perjuicios que beneficios.

Entrando en el tratamiento de las hemorragias uterinas excesivas, lo primero que hay que tener en cuenta es el problema de Ia anemia, pues hay casos en que por causas puramente funcionales pueden producirse hemorragias verdaderamente depletivas. Estos casos se tratarán como emergencias, por restitución de la sangre perdida y control de la hemorragia, como lo veremos más adelante.

Cuando la hemorragia excesiva está asociada con ciclos ovu. iatorios, tenemos en general tres tipos diferentes: a) La hemorragia die la ovulación, intermenstrual o bifásica; b) La derivada de hipoprogestinismo, que produce una fase luteal corta y por consiguiente una falta de fase luteal completa y mala descamación endometrial, y c) La ocasionada por descamación o desprendimiento irregular del endometrio.

La hemorragia de ovulación es excepcionalmente excesiva, peIo a veces puede durar tres o cuatro días y simular una men truación ordinaria, constituyendo entonces la llamada hemorragia bifásica. Es muy fácil de diferenciar la hemorragia de ovulación de la menstruación, mediante la curva de temperatura basal, pues si la sangre se presenta en el momento del alza térmica ovulatoria, será hemorragia de ovulación, y si se presenta en el momen. 
to del descenso térmico menstrual, será hemorragia de menstrua ción. La biopsia de endometrio practicada durante el período hemorrágico también hace la diferencia, pues en la hemorragia do ovulación la paciente sangrará de un endometrio proliferativo es. trogénico, y en la menstruación sangrará de un endometrio progestacional, puesto que ha habido ovulación.

Cuando la hemorragia de ovulación requiera tratamiento, se hará con estrógeno y progesterona simultáneos por vía oral, comenzando unos dos dias antes de la ovulación para evitar así el descenso estrogénico post-ovulatorio, que es al que ocasiona la hemorragia, y continuando la medicación por unos 14 días, para sus. penderla unos 4 días antes de la fecha en que se espera o se desea que aparezca la nueva menstruación. En muchas de estas pacienLes el tratamiento $\lesssim \in$ hace porque la hemorragia de ovulación imwide la actividad coital en el momento preciso en que debe realizarse por buscar un embarazo, y ésta es la razón por la cual la administración de estrógenos sólo debe iniciarse unos dos días an. Les de la ovulación, para que los estrógenos no vayan a interfeiir con la ovulación.

Las pacientes con acortamiento de la fase luteal no deben recibir tratamiento, a no ser que presenten problema de infertiiidad por maia receptividad del endometrio para el huevo fecundado, a causa de la actividad progestacional defíciente. En estos casos el tratamiento se hará con estrógenos y progesterona, partiendo del día de la ovulación y prolongándolo por 10 a 14 días. Hay que estar atentos a la aparición de la próxima menstruación, pues si ésta se tarda más de 4 días después de suspendido el tratamiento, debe presumirse la existencia de embarazo y continua: sa terapia, ya que si el cuerpo lútero era insuficiente para produ. cir un buen endometrio progestaciorial, es de pensarse que deba también ser insuficiente para albergar el embrión, y entonces la terapéutica sustitutiva debe prolongarse hasta que el sistema co. vio-piacentario pueda suplir totalmente la función deficiente de! «uerpo amarillo.

En cuanto a la descamación irregular del endometrio, creo que \& tratamiento de elección as el curetaje cuidadoso practicado el 4 momento correspondiente a la regla, seguido inmediatamente ciespués de una terapéutica cíclica estrógeno-progestorona por dos a tres ciclos consecutivos. En cada ciclo se darán estrógenos por ajpacio de 20 días, y en los diez últimos días de estos 20 , progesterona, en tal forma que al terminar los 20 días de medicación se 
suspenden bruscamente las dos hormonas. En esta forma, la menstruación debe aparecer unos cuatro días después de suspendida la medicación. Después de dos o tres ciclos artificiales, muy segu. samente seguirán períodos normales y se acabará el problema de ia descamación irregular del endometrio.

Para terminar con las hemorragias asociadas a ciclos ovulatorios, debemos recordar que hay que hacer el diagnóstico correcto de si la hemorragia es o no funcional, pues no es tara la concomitancia de los ciclos ovulatorios con lesiones ginecológicas (pólipos, miomas, anexitis, etc.), o con lesiones sanguíneas (púrpura hemorrágica), etc., que pueden ocasionar hemorragias abundantes provenientes de un endometrio progestacional.

Los casos más frecuentes de hemorragia uterina funcional excesiva, prolongada y frecuente, están asociados con ciclos anovulatorios. Estas pacientes no descaman completamente el endometrio por falta de fase de secreción adecuada (ausencia de cuerpo Iúteo, pues no ha habido ovulación), y entonces tiende a injertar nuevas fases de crecimient, sobre endometrio insuficientemente descamado, produciéndose la hiperplasia endometrial, ya que el antiguo endometrio insuficientemente descamado actúa como un gran pólipo sésil y agrega su propio ritmo de crecimiento al del nuevo ciclo. Estas pacientes pueden sangrar a partir de un endometrio estrogénico precoz o tardío, o de un endometrio hiperplásico, que es el caso más frecuente.

Colocados frente a uno de estos casos, la terapéutica debe dirigirse a tres objetivos primordiales: a) La hemostasis inmediata, para evitar que la paciente continúe perdiendo sangre; b) evitar que se repita el episodio de hemorragia excesiva, y c) Después de suspender el tratamiento, lograr que la paciente tenga ciclos nor. males espontáneos, y ojalá ovulatorios.

Para lograr el primer objetivo, o sea la hemostasis inmediata, creemos que el mejor procedimiento es el curetaje cuidadoso que aconsejamos practicar de rutina en las mujeres mayores de veinticinco años, pues además de que sirven para remover el tejido hemorrágico, sirve también para descartar de una vez la posibilidad de una lesión neoplásica endometrial. No usamos este sistema en las niñas, para las cuales hay otros procedimientos. Creo que el curetaje es el mejor de los hemostáticos, pero de nada vale practicarlo si la paciente no recibe una terapéutica complementaria que evite que 10 o 12 días después del raspado vuelva a sangrar. La hemostasis es sólo el comienzo. Aconsejo el curetaje 
en mujeres menores de veinticinco años cuando la hemorragia es de tal abundancia que liega a convertirse en emergencia y no se pueden esperar los tres o cuatro dias que se requieren para hacer la hemostasis con estrógenos. En estos casos debe hacerse el curetaje y reponer inmediatamente la sangre perdida.

Cuando la hemorragia no es depletiva se puede dar la tera_ péutica estrogénica con el fin de elevar los niveles de estrógenos y procurar asi una nueva fase de crecimiento endometrial, que por sí misma suspenderá la hemorragia. El estrógeno que preferimos para estos casos es el Premarin o Ayerogen, del cual pueden requerirse hasta 15 miligramos al día y aun más, en los casos de hiperplasia. Una vez conseguida la hemostasis, sea por la administración de estrógenos o por el curetaje, no se puede dejar a la paciente sin medicación, sino que deben continuarse las mismas dosis hemostáticas por espacio de 20 dias después de lograda la nemostasis, para posponer el nuevo episodio de hemorragia, darie a la paciente tiempo de restablecer sus niveles de hemoglobina, y procurar el desarrollo de ciclos regulares normales. Si se reduce significativamente la dosis de estrógenos que fue necesaria para procurar la hemostasis, lo que haremos será una baja brusca de estrógenos en la sangre, es decir, lo mismo que ocurre espontáneamente cuando se sucaden las hemorragias. Por esto es que hay que continuar con las mismas dosis. Es claro que en los diez días finales de los 20 en que la paciente está recibiendo estrógenos, debe recibir cantidades adecuadas de progesterona para que el endometrio descame normalmente, pues como la terapia estrogénica inhıbe la ovulación, no habrá cuerpo lúteo y entonces hay que suplir artificialmente la hormona progestacional. Las dosis de progesterona serán tanto más altas cuanto lo sean las dosis de estrógenos empleados.

El sistema de hemostasis inmediata en las pacientes muy jóvenes, o vírgenes, o en las que por cualquiera razón no se puede practicar el curetaje, es la administración intravenosa de estrógenos (Ayerogen). Con este procedimiento se logra la hemostasis en el curso de unas seis horas después de aplicada la inyección. El mecanismo de acción no es muy claro, pues no se puede pretender que los estrógenos administrados por vía intravenosa vayan a ocasionar una regeneración endometrial en tan corto tiempo, pero el hecho es que la hemostasis se produce. Parece que existe una acción especifica de los estrógenos sobre la coagulación sanguinea, que en parte incluye el aumento de ciertas globu- 
nas y de la cantidad de protrombira, y la disminución de la an. titrombina, hasta dcnde se sabe actualmente.

Debe aavertirse nuevamente que no es en ningún modo ra. zonable suministrar los estrógenos intravenosos, suspender la hemorragia y descontinuar el tratamiento, pues la paciente volvera entonces a sangrar. Los estrógenos intravenosos deben continuarse con una a tres inyecciones diarias por dos o tres días, so gún las necesidades, y desde el primer dia instituír la terapéuti ca con estrógenos orales, para que cuando se descontinúe el efec. to de la medicación endovenosa, la paciente tenga un nivel de es. trógenos orales suficiente para manterer la regeneración endo. metrial. Así, pues, la medicación estrogénica oral debe mantene: se por espacio de 20 dílas, usando progesterona en los 10 últimos días, en la forma enunciada anteriormente.

En los casos de hemorragias de pequeña intensidad pero de duración irregular y que se hacen a vece: casi permanentes, el tratamiento de elección sería dar estrógenos asociados con progesterona, ambos orales, por 10 dias para provocar al suspender los la descamación endometrial, y poder entonces iniciar los ei. clos eitrógeno-progesterona complatos por dos o tres meses, con la esperanza de que estas pacientes reinicien ciclos menstruales normales y espontáneos.

También puede lograrse una hemostaミis uterina no inmediata con progesterona. Al suministrar 25 miligramos de progesterona en aceite intramuscular cada tercer dia, por tres a cinco dosis, se procura la descamación del endometrio sangrante. Es claro que ia paciente puede sangrar durante el tiempo en que esté recibiendo ia progesterona, pero al suspender ésta se producirá la descamación del endometrio y dehe suspenderse la hemorragia. Por esta razón digo que es un sistema de hemostasis tarda.

La progesterona que se usa en los ciclos artificiales puede ser administrada por vía oral (1.5 miligramos diarios por 10 dias), 0 ror vía parenteral. Si se usan inyecciones de progesterona en acei-te, se darán 25 a 50 miligramos por vía intramuscular cada ter. cer día por cinco dosis. Si se usan progesteronas de acción retardada (depot), bastará generalmente una inyección de 250 miligramos aplicada el día décimo de la terapia estrogénica, pues se supone que esta medicación es efectiva por un tiempo de 10 a 12 días. Se obtienen también excelentes reisultados con el uso de los derivados de la nortestosterona, la ethisierona y algunos derivados de la 17-alpha-hidroxiprogesterona, nuevos progestínicos de 
uso oral y de una actividad progestacional no conocida antes. Las dosis serán de 5 a 10 miligramos al día, según la cantidad de es.. trógenos empleada.

Sería un error si les dijera que necesariamente deben usarse estrógenos en los tratamientos cíclicos de la hemorragia funcional. Si se tiene una paciente en la cual por el curetaje se demuestra que está sangrando de un endoometrio hiperplásico, no hay razón ninguna para que el curataje sea necesariamente seguido ce terapia estrogénica. En estos casos bastará con dar 14 dias después del curetaje 25 miligramos de progesterona en aceite $\mathbf{I}$. $\mathbf{M}$. cada tercer día por cinco dosis, y repetirlo en la misma forma por tres o cuatro ciclos. O una inyección de progesterona de acción retardada de 250 miligramos, también 14 días después del cu. ietaje, o iniciar a los 14 días la administración oral de progesterona por 10 días. Lo importante es repetir el ciclo de progesterona por unos cuatro meses consecutivos para obtener una buena descamación endometrial. La mayoría de estas pacientes, al suspender el tratamiento, tendrán ciclos perfectos de duración normal, y usualmente ovulatorios.

Para resumir: la hemorragia funcional uterina se diagnostica por exclusión de todos los factores médicos, ginecológicos o endocrinológicos. Se puede obtener una hemostasis apropiada y rápida por medio de estrógenos orales o intravenosos, o por el curetajo, dependiendo la elección del sistema del grado de la anemia $y$ de la intensidad de la hemorragia. A toda mujer mayor de veinticinco años con hemorragia uterina excesiva, debe practicársele dilatación y curetaje para diagnóstico como para hemostasis. La hemostasis tardia puede también obtenerse con progesterona, en la forma enunciada. Una vez obtenida la hemostasis, se continuará el tratamiento cíclico con estrógenos y progesterona, o con progesterona sola, según los casos en la forma descrita. No encuentro justificación racional para el uso de andrógenos en el trata_ miento de la hemorragia funcion $x$ l uterina. Espero haber podido justificar ante ustedes la lógica y razón de los tratamientos propuestos.

En fin, no se pueden dogmatizar los tratamientos propuestos, y catalogarlos y describirlos en forma de términos absolutos. Lo importante es saber qué es lo que se quiere hacer, qué efecto van a producir las drogas prescritas, y cuáles deben ser las dosis. No todas las personas responden a los tratam.entos en la misma forma y habrá por ejemplo pacientes que sangran antes de lo espe- 
rado, lo cual puede ser porque no están recibiendo los estrógenos en dosis suficientes, o porque están recibiendo demasiada progesterona. El tratamiento deberá adaptarse entonces a estos casos inđividuales.

Espero haberles dado una idea clara de este tratamiento con. servador, que en las mujeres jóvenes permitirá la iniciación o el retardo de la ovulación en un $65 \%$ de los casos, aproximadamente. Es un sistema que me parece ideal por sus claras bases fisiológicas, porque resuelve la emergencia, mejora a la paciente sin afectar la función ovárica, y usualmente da por resultado mujeres con ciclos ovulatorios, aptias para concebir. Los que tuvieron oportunidad de conocer estos problemas en los días anteriores a la existencia de drogas estrogénicas y progestínicas activas, tendrán ccasión de sufrir con el tratamiento de estas hemorragias, con los curetajes repetidos indefinidamente a mujeres jóvenes, las transfusiones, los taponamientos, la irradiación, o en muchas ocasiones la histerectomia. Es muy grato poder disponer hoy en día de un tratamiento racional y efectivo para estas condiciones.

Me complace decir que si algún aporte he hecho a la endo. crinología en el curso de treinta años de dedicación a estos est?: dios, es esta forma de tratamient?. 